\title{
Efeito da técnica de inserção e do tempo de fotoativação na dureza de resinas compostas bulk fill
}

\author{
João Felipe Besegato Departamento de Odontologia Restauradora, Faculdade de Odontologia de Araraquara, \\ Universidade Estadual Paulista (Unesp), Araraquara, SP, Brasil - Débora de Castro Costa Petrin Departamento \\ de Odontologia Restauradora, Universidade Estadual de Londrina (UEL), Londrina, PR, Brasil • Giovana Cazarim da \\ Costa Departamento de Odontologia Restauradora, Universidade Estadual de Londrina (UEL), Londrina, PR, Brasil \\ - Lucas Fernando de Oliveira Tomaz Ferraresso Departamento de Odontologia Restauradora, Universidade Estadual \\ de Londrina (UEL), Londrina, PR, Brasil - Márcio Grama Hoeppner Departamento de Odontologia Restauradora, \\ Universidade Estadual de Londrina (UEL), Londrina, PR, Brasil
}

RESUMO | Objetivos: Avaliar o efeito da técnica de inserção e do tempo de fotoativação na dureza Vickers de resinas compostas bulk fill (RCBF). Materiais e método: RCBF de alta viscosidade Opus Bulk Fill (OBF) e de baixa viscosidade Opus Bulk Fill Flow (OBFF) foram inseridas em matrizes cilíndricas com altura e diâmetro interno de $4 \mathrm{~mm}$, e divididas em seis grupos (G), de acordo com a técnica de inserção ( $\mathrm{I}^{1}-$ incremento único de $4 \mathrm{~mm}$ e $\mathrm{I}^{2}-$ dois incrementos de $2 \mathrm{~mm})$ e do tempo de fotoativação de cada incremento com irradiância de $1000 \mathrm{~mW} / \mathrm{cm}^{2}\left(\mathrm{~T}^{1}-20\right.$ segundos e $\mathrm{T}^{2}-40$ segundos): $\mathrm{G} 1$ - OBFI ${ }^{1} \mathrm{~T}^{1}, \mathrm{G} 2-\mathrm{OBFI}^{2} \mathrm{~T}^{1}, \mathrm{G}_{3}-\mathrm{OBFI}^{1} \mathrm{~T}^{2}, \mathrm{G}_{4}-\mathrm{OBFI}^{2} \mathrm{~T}^{2}, \mathrm{G}_{5}-\mathrm{OBFF} \mathrm{OBFI}^{2} \mathrm{~T}^{1} \mathrm{e}$ $\mathrm{G} 6-\mathrm{OBFF}+\mathrm{OBFI}^{2} \mathrm{~T}^{2}$. A dureza foi avaliada na superfície topo e base das amostras. ANOVA dois critérios seguido de pós-teste de Tukey foram utilizados em nível de significância de 5\%. Resultados: Não houve diferença na dureza da superfície topo das amostras, independentemente da técnica de inserção e do tempo de fotoativação. Entretanto, na superfície base, houve diferença entre as técnicas de inserção e os tempos de fotoativação, exceto entre G3 e G4. Todas as amostras apresentaram redução significativa de dureza na base em relação ao topo. Conclusão: Pode-se concluir que a fotoativação com menor tempo de exposição deve ser considerada com cautela, pois resultou na redução da dureza na área mais distante da fonte emissora da luz. OBFF apresentou dureza menor que OBF, independentemente do tempo de fotoativação.

ABSTRACT | Effect of filling technique and light-curing exposure time on the hardness of bulk-fill resin composites - Objectives: This study evaluated the effect of filling techniques and light-curing time on Vickers hardness of bulk fill composite resins (BFCR). Methods: BFCR high viscosity Opus Bulk Fill (OBF) and low viscosity Opus Bulk Fill Flow (OBFF), were placed in cylindrical samples of poly methyl methacrylate with height and internal diameter of $4 \mathrm{~mm}$, and randomly assigned into six groups (G) according to the filling technique ( $\mathrm{I}^{1}$-single increment of $4 \mathrm{~mm}$ thickness and $\mathrm{I}^{2}-$ increment of $2 \mathrm{~mm}$ thickness) and light-curing time of each increment with an irradiance of $1000 \mathrm{~mW} / \mathrm{cm}^{2}\left(\mathrm{~T}^{1}-20\right.$ seconds and $\mathrm{T}^{2}-40$ seconds): $\mathrm{G} 1-\mathrm{OBFI}^{1} \mathrm{~T}^{1}, \mathrm{G} 2-\mathrm{OBFI}^{2} \mathrm{~T}^{1}, \mathrm{G}_{3}-\mathrm{OBFI}^{1} \mathrm{~T}^{2}, \mathrm{G}_{4}-$ $\mathrm{OBFI}^{2} \mathrm{~T}^{2}, \mathrm{G} 5-\mathrm{OBFF}+\mathrm{OBFI}^{2} \mathrm{~T}^{1}$ e G6 $-\mathrm{OBFF}+\mathrm{OBFI}^{2} \mathrm{~T}^{2}$. Hardness was measured in the top and bottom surface of each sample. Twoway ANOVA followed by Tukey's post-test were used for analysis with a significance level of $5 \%$. Results: There was no difference in hardness on the top surface, regardless of the filling technique and light-curing exposure time used. However, in the bottom surface differences were observed regarding the filling technique and light-curing time, except between the G3 and G4 groups. All samples showed significant hardness reduction at the bottom compared to the top surface. Conclusions: The lowest light-curing time (20 seconds) must be considered with caution, as it resulted in the reduction of hardness in the area farther from the light source. The OBFF exhibited lower hardness than OBF, despite of the light-curing exposure time.

AUTOR CORRESPONDENTE 


\section{INTRODUÇÃO}

A resina composta (RC) é o material de escolha dos cirurgiões dentistas para a restauração direta de dentes anteriores e posteriores..$^{1-3}$ A otimização da conversão de monômeros em polímeros e, por consequência, as propriedades físicas, mecânicas e biológicas da RC são dependentes da utilização de aparelho fotoativador com irradiância adequada. ${ }^{4,5}$

Para garantir a propagação da luz em toda a massa de RC inserida na cavidade, é indicada a técnica incremental, que consiste na inserção e fotoativação de incrementos de RC de, no máximo, $2 \mathrm{~mm}$ de espessura.$^{6-9}$ Entretanto a inserção de vários incrementos na restauração de cavidades extensas e profundas em dentes posteriores, além de consumir mais tempo clínico, aumenta a probabilidade de contaminação durante o procedimento, ${ }^{10-11} \mathrm{de}$ incorporação de bolhas no interior da $\mathrm{RC}^{12}$ e de detritos entre as camadas de RC. ${ }^{13}$

Assim, para simplificar a técnica restauradora e diminuir o tempo clínico, surgiram as RC do tipo bulk fill (BF) ou de preenchimento único. ${ }^{14-16}$ Maior profundidade de polimerização, menor contração e menor tensão na interface dente-restauração ${ }^{17,18}$ sustentam a inserção das RCBF em incremento único de 4 a $5 \mathrm{~mm}$ de espessura para a restauração de dentes posteriores.

As RCBF flow de baixa viscosidade, quando utilizadas para a restauração da dentina, requerem o recobrimento de um incremento de $\mathrm{RC}$ convencional, de 1,5 a $2 \mathrm{~mm}$, para finalizar a restauração. ${ }^{19}$ Por sua vez, as RCBF de alta viscosidade, embora indicadas para preenchimento cavitário num único incremento, apresentam maior deformidade em áreas de grandes esforços mastigatórios e maior probabilidade de fratura da restauração. ${ }^{20}$ Dessa forma, a inserção de um incremento de RCBF de baixa viscosidade, antes do incremento de RCBF de alta viscosidade na técnica do sanduíche, melhora o selamento marginal ${ }^{21} \mathrm{e}$ a adaptação interna à dentina. ${ }^{22}$

Para mensurar os valores de dureza da $\mathrm{RC}$ em relação ao protocolo de inserção e fotoativação, independentemente da proposta dos fabricantes, é relevante a realização de testes laboratoriais. ${ }^{23}$ Assim, o objetivo deste estudo foi avaliar o efeito da técnica de inserção e do tempo de fotoativação na dureza Vickers (DV) de RCBF. A hipótese nula testada foi que o protocolo de inserção e o tempo de fotoativação de polimerização não influenciam na DV das RCBF.

\section{MATERIAIS E MÉTODO}

\section{Delineamento experimental}

Este estudo in vitro, cego para o estatístico e randomizado quanto à sequência da confecção das amostras, teve como variáveis independentes a técnica de inserção e o tempo de fotoativação do incremento de RCBF. A variável dependente foi a DV no topo e na base das amostras obtidas com duas RCBF: Opus Bulk Fill Flow (OBFF) de baixa viscosidade e Opus Bulk Fill (OBF) de alta viscosidade (Tabela 1 ).

Tabela 1 | Especificações das RCBF utilizadas.

\begin{tabular}{|c|c|c|c|c|}
\hline Resina Composta & Fabricante & Composição* & $\begin{array}{l}\text { Quantidade de } \\
\text { carga (\% peso) }\end{array}$ & Cor \\
\hline $\begin{array}{l}\text { Opus Bulk Fill } \\
\text { (OBF) }\end{array}$ & FGM, Joinville, SC, Brasil & $\begin{array}{l}\text { Monômeros uretanadimetacrílicos, Estabilizantes, Fotoiniciadores } \\
\text { Coiniciadores, Cargas inorgânicas de dióxido de silício (sílica) } \\
\text { silanizado, Pigmentos }\end{array}$ & 79 & $\mathrm{~A} 2$ \\
\hline $\begin{array}{l}\text { Opus Bulk Fill Flow }{ }^{\mathrm{T}} \\
\text { (OBFF) }\end{array}$ & FGM, Joinville, SC, Brasil & $\begin{array}{l}\text { Monômeros uretanadimetacrílicos, Estabilizantes, } \\
\text { Canforoquinona, } \\
\text { Coiniciadores, } \\
\text { Cargas inorgânicas de dióxido de silício (sílica) } \\
\text { silanizado e Pigmentos }\end{array}$ & 68 & A2 \\
\hline
\end{tabular}

*Informações disponibilizadas pelos respectivos fabricantes. 
Conforme ilustrado na Figura 1, foram empregadas duas técnicas de inserção: incremento único de $4 \mathrm{~mm}$ de espessura ( $\mathrm{G}_{1}$ e $\left.\mathrm{G}_{3}\right)$ ou dois incrementos de $2 \mathrm{~mm}$ de espessura (G2, G4, G5 e G6). Em relação ao tempo de fotoativação, dois tempos foram testados: 20 e 40 segundos para cada incremento de RCBF inserido no interior das matrizes, de acordo com os grupos de estudo.

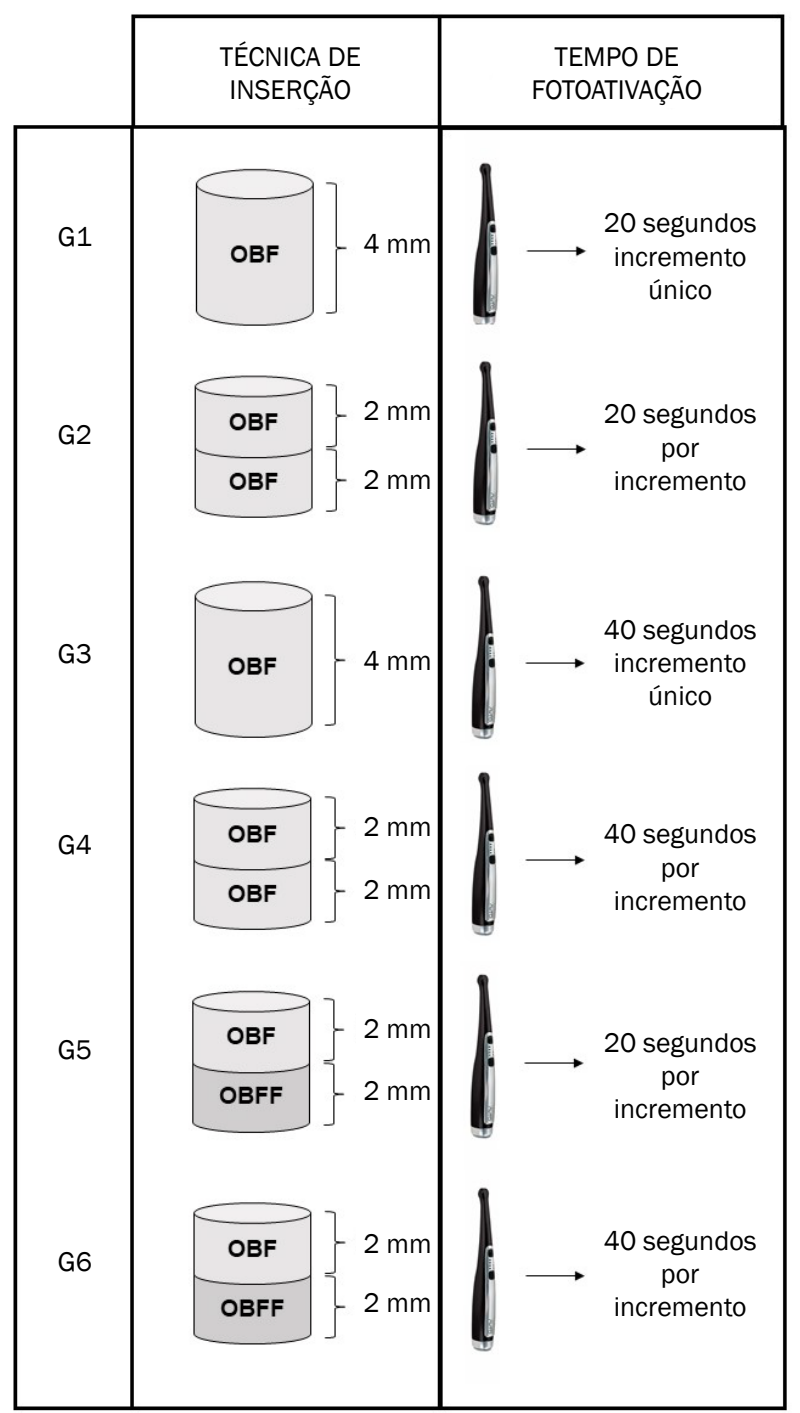

Figura 1 | Desenho esquemático dos grupos de estudo.

\section{Confecção das matrizes e amostras}

As matrizes foram confeccionadas com auxílio de torno mecânico (Nardini 350, Americana (SP), Brasil) com dimensão e altura interna de
4 x 4 mm. O material utilizado para a confecção das matrizes foi o polimetilmetacrilato (PMMA) em cor semitransparente,,${ }^{24}$ com o intuito de reproduzir as situações clínicas de maneira mais fidedigna.

Os incrementos de RC OBF foram inseridos no interior de cada matriz com auxílio de uma espátula (Suprafill N1, SSWhite Duflex, São Cristóvão, RJ, Brasil), enquanto os incrementos de RC OBFF foram inseridos com o auxílio de uma ponteira aplicadora fornecida pelo respectivo fabricante. Quando do preenchimento em incremento único ( $\mathrm{G}_{1}$ e $\mathrm{G}_{3}$ ) e quando da inserção do segundo incremento (G2, G4, G5 e G6), uma lamínula de microscopia (Labor Import, Guarulhos, SP, Brasil) foi posicionada sobre a matriz e aplicada leve pressão digital para remover o excesso de material e inibir o contato com o oxigênio durante a polimerização. ${ }^{25}$

\section{Fotoativação das RCBF}

Todos os incrementos de RCBF foram fotoativados com um aparelho fotopolimerizador à base de diodos emissores de luz (LEDs) (VALO Cordless, Ultradent Products, South Jordan, UT, USA) de amplo espectro (comprimento de onda entre 395 e $480 \mathrm{~nm}$ ), com irradiância de $1.000 \mathrm{~mW} / \mathrm{cm}^{2}$ (modo standard) e tempo de exposição de 20 e 40 segundos, de acordo com os grupos de estudos. $\mathrm{O}$ aparelho foi posicionado sobre a lamínula de vidro e estabilizado com auxílio de um braço mecânico, a fim de evitar oscilações do aparelho e interferências na irradiância emitida durante o tempo de exposição.

Durante o experimento, utilizou-se um potenciômetro (Nova P/N 7Z01500, Ophir Optronics Solutions Ltd, Har Hotzvim, Jerusalém, Israel) acoplado a um sensor de mensuração de energia (PD300 P/N 7Zo2410 Ophir Optronics Solutions Ltd, Har Hotzvim, Jerusalém, Israel) para verificar a irradiância emitida pelo aparelho fotoativador $\left(\sim 1060 \mathrm{~mW} / \mathrm{cm}^{2}\right)$. 


\section{Análise de dureza Vickers (DV)}

Imediatamente após o preenchimento completo da matriz e fotoativação da RC, a DV de cada amostra foi avaliada com o auxílio de um aparelho microdurômetro (HM-100, Mitutoyo Sulamericana LTDA, SP, Brasil) endentador de diamante na forma de pirâmide reta de base quadrada. Foram utilizadas carga de $100 \mathrm{~g}$ e tempo de 15 segundos $^{26}$ para a realização de três endentações na superfície topo (o mm) e três na base (4 mm) das amostras. A média aritmética das três endentações para cada amostra em cada superfície foi calculada para a análise estatística dos dados.

\section{Análise estatística}

A normalidade dos dados foi verificada por meio do teste de Shapiro-Wilk ( $\mathrm{p} \geq 0,05)$. Aplicou-se o teste paramétrico de ANOVA dois fatores, seguido de pós-teste de Tukey para análise de variância da dureza Vickers nas superfícies topo e base das amostras, de acordo com as diferentes técnicas de preenchimento. As análises foram realizadas utilizando o software BioEstat 5.3 (Instituto de Desenvolvimento Sustentável Mamirauá, Tefé, AM, Brasil), em nível de significância de 5\%.

\section{RESULTADOS}

Não houve diferença na dureza da superfície topo das amostras de RCBF, independentemente da técnica de inserção e do tempo de fotoativação da polimerização ( $p>0,05)$. Entretanto houve diferença na superfície base ( $\mathrm{p}<0,05)$, exceto entre os grupos $\mathrm{G}_{3}$ (OBF 40 s incremento único) e $\mathrm{G}_{4}$ (OBF $40 \mathrm{~s}$ dois incrementos) ( $\mathrm{p}>0,05)$. As amostras de todos os grupos apresentaram redução significativa de dureza na base em relação ao topo $(\mathrm{p}<\mathrm{0,05})$, sendo que G4 apresentou maior percentual de DV na superfície base em relação ao topo (70,99\%) e G5, o menor percentual (27,46\%). Além disso, a técnica de inserção envolvendo dois incrementos de RCBF (G2, G4, G5 e G6) resultou em aumento da DV ( $\mathrm{p}$ < 0,05) na superfície base em comparação à mesma superfície dos grupos de preenchimento único (G1 e G3) (Tabela 2).

Tabela 2 || Média ( \pm desvio padrão) da dureza Vickers (VHN) no topo e base das amostras e percentual de variação de DV entre as superfícies $(n=10)$.

\begin{tabular}{|c|c|c|c|}
\hline \multirow[b]{2}{*}{ Grupo } & \multicolumn{3}{|c|}{ Profundidade } \\
\hline & Topo & Base & $\begin{array}{l}\text { Percentual de } \\
\text { DV da base em } \\
\text { relação ao topo (\%) }\end{array}$ \\
\hline G1 & $52,83 \pm 1,99$ A,a & $26,70 \pm 1,59^{A, b}$ & 50,53 \\
\hline G2 & $52,03 \pm 1,14^{\mathrm{A}, \mathrm{a}}$ & $31,42 \pm 1,21^{B, b}$ & 60,38 \\
\hline G3 & $53,46 \pm 0,24^{A, a}$ & $36,19 \pm 2,36^{c, b}$ & 67,69 \\
\hline G4 & $53,41 \pm 1,200^{A, a}$ & $37,92 \pm 1,46^{c, b}$ & 70,99 \\
\hline G5 & $52,32 \pm 0,30^{A, a}$ & $14,37 \pm 0,59^{\mathrm{D}, \mathrm{b}}$ & 27,46 \\
\hline G6 & $53,14 \pm 1,01^{\mathrm{A}, \mathrm{a}}$ & $21,11 \pm 1,12 \mathrm{E}, \mathrm{b}$ & 39,72 \\
\hline
\end{tabular}

Letras minúsculas diferentes na linha significam diferença estatística significante $(p<0,05)$.

Letras maiúsculas diferentes na coluna significam diferença estatística significante $(p<0,05)$.

\section{DISCUSSÃO}

Neste estudo, considerando os valores de DV obtidos na superfície base, a hipótese nula testada foi rejeitada, pois houve diferença entre os grupos $(\mathrm{p}<\mathrm{o,05})$. Contudo isso não foi observado na superfície do topo das amostras, aceitando-se, assim, a hipótese nula ( $\mathrm{p}>0,05)$. Para G1, G2, G5 e G6, os valores de dureza obtidos na superfície base foram influenciados pelos fatores tempo de exposição e irradiância, bem como pelo volume de RCBF fotoativado.

Quanto à composição, por também ser um material restaurador de base monomérica, as RCBF se assemelham às RC convencionais. Porém a utilização clínica para a restauração de dentes posteriores por meio da técnica de inserção e fotoativação de incremento único, com espessura entre 4 a $5 \mathrm{~mm}$, foi possível a partir de mudanças na composição química, como: acréscimo de fotoiniciadores mais reativos, ${ }^{8,27}$ aumento da translucidez ${ }^{28,29}$ e diminuição do conteúdo inorgânico. ${ }^{30}$ Embora essas modificações tenham por objetivo reduzir o tempo clínico do procedimento 
restaurador, a inserção de incremento único de $4 \mathrm{~mm}$ de OBF influenciou na diferença dos valores de DV na superfície base.

Para incrementos de RC de $2 \mathrm{~mm}$, o valor de dureza observado na superfície base deve ser o equivalente a $80 \%$ do valor de dureza obtido na superfície topo..$^{20,31}$ Neste estudo, para o incremento de $4 \mathrm{~mm}$ de profundidade, essa relação de variação do percentual de dureza entre as superfícies base e topo não foi observada em nenhum dos grupos (Tabela 2). Portanto a inserção de fotoativação de incremento único de $4 \mathrm{~mm}$ da OBF deve ser reavaliado para garantir a longevidade da restauração. Vale ressaltar que, além de comprometer as propriedades mecânicas das RCBF, a conversão insuficiente resulta em monômeros residuais que, consequentemente, aumentam a citotoxicidade dos compósitos..$^{32-34}$

Neste estudo, o tempo de exposição foi empregado para cada incremento de RCBF. Em G2, G4, G5 e G6, o primeiro incremento de $\mathrm{RC}$ recebeu maior quantidade de fótons (maior tempo de exposição) em comparação aos grupos de preenchimento único (G1 e G3), o que pode ter contribuído para os maiores valores de dureza na superfície base dos respectivos grupos, além da espessura dos incrementos (Tabela 2).

A redução significativa dos valores de dureza na superfície base, em relação à superfície topo, pode também ter sido influenciada pela propriedade óptica da OBF de absorção e atenuação da propagação da luz pelo material. ${ }^{35}$ Comparativamente à $\mathrm{OBF}$, a $\mathrm{OBFF}$ apresenta menor quantidade de partículas inorgânicas e maior concentração de material orgânico (Tabela 1). Esses fatores podem ter contribuído para os baixos valores de dureza observados em G5 e G6. Mesmo apresentando maior translucidez, a fotoativação de incremento de $2 \mathrm{~mm}$ de espessura de OBFF não foi suficiente para atenuar a perda dos valores de dureza na superfície base das amostras (Tabela 2), ratificando estudos de Hahnel et al. ${ }^{36}$ e Besegato et al. ${ }^{37}$

Reproduzir as condições bucais de maneira fidedigna em estudos in vitro é um desafio metodológico. O diâmetro, a cor e o tipo de material utilizado na confecção da matriz afetam a profundidade de cura dos materiais a serem avaliados..$^{38}$ De acordo com os resultados deste estudo prévio, matriz de maior diâmetro e confeccionada com material semitransparente promove maior profundidade de polimerização. ${ }^{38}$ Além do mais, matriz semitransparente e branca pode ser mais eficaz para simular as propriedades ópticas da estrutura dentária, quando comparada à matriz metálica e opaca. ${ }^{39}$ Com o intuito de superar essas limitações, as matrizes utilizadas neste estudo foram confeccionadas com PMMA semitransparente.

É importante ressaltar que este estudo avaliou apenas uma variável (dureza). Para uma avaliação mais adequada em relação à caracterização dos materiais e do efeito das técnicas empregadas, métodos de análise adicionais são imprescindíveis. Além disso, o baixo valor de dureza para a OBFF já era esperado, considerando sua composição. No entanto, apesar desses baixos valores observados, o uso de RCBF de baixa viscosidade previamente à inserção de uma RC de alta viscosidade está indicado para melhorar a adaptação às paredes internas dentinárias, ${ }^{28}$ dissipar as forças mastigatórias ${ }^{20} \mathrm{e}$ minimizar o risco de fratura da restauração. ${ }^{40}$ Assim, os resultados deste estudo devem ser interpretados com cautela e considerando o objetivo da técnica utilizando RCBF de baixa viscosidade.

O tempo de exposição e a irradiância emitida pelo aparelho fotoativador são essenciais para a eficácia da fotopolimerização. Neste estudo, utilizou-se um aparelho à base de LED. Embora lâmpadas LED não apresentem colimação do feixe de luz comparandose aos lasers, o equipamento VALO apresenta lentes difusoras que propiciam maior colimação do feixe de luz e mantém a intensidade de luz em sua ponta ativa de $10 \mathrm{~mm}$ de diâmetro. ${ }^{41} \mathrm{Em}$ teoria, a colimação seria uma vantagem, pois garante que a luz irradiada da fonte emissora chegue de forma mais uniforme à superfície a ser fotoativada, diminuindo sua dispersão. 
Somado a isso, o diâmetro da ponta fotoativadora, o perfil do feixe e o espectro de emissão da luz afetam a qualidade da fotoativação de RCBF. ${ }^{42}$ No entanto, neste estudo, a colimação do feixe de luz e as demais características do aparelho fotoativador não foram suficientes para minimizar a redução dos valores de dureza para todos os grupos. Dessa forma, é possível inferir que a perda de irradiância acontece principalmente em função do aumento da distância entre a fonte de luz e a superfície da RC.

A irradiância testada neste estudo foi de $1.000 \mathrm{~mW} / \mathrm{cm}^{2}$ (modo standard), tendo variações no tempo de exposição. G1 e G2, com 20 segundos de fotoativação por incremento, apresentaram menores valores que $\mathrm{G}_{3}$ e $\mathrm{G} 4$, com 40 segundos por incremento, ratificando que o tempo de exposição influencia na conversão e na dureza da RC. ${ }^{37}$ Diante dos resultados encontrados, sugere-se que o profissional tenha prudência quanto às orientações do fabricante a respeito do tempo de fotoativação, da irradiância recomendada e da espessura do incremento para o alcance de adequada polimerização de toda a extensão do incremento de material resinoso.

\section{CONCLUSÕES}

Considerando as limitações e os resultados obtidos neste estudo in vitro, pôde-se concluir que a técnica de inserção e o tempo de exposição influenciaram na dureza da superfície base das amostras, o que não ocorreu na superfície topo. A fotoativação com menor tempo de exposição deve ser considerada com cautela, pois resultou em maior redução da dureza na área mais distante da fonte emissora da luz. Adicionalmente, OBFF apresentou dureza menor que a OBF, independentemente do tempo de exposição.

\section{AGRADECIMENTOS}

Os autores agradecem à FGM Produtos Odontológicos LTDA e à Ultradent Products Inc. pela doação dos materiais utilizados nesta pesquisa.

\section{CONFLITO DE INTERESSE}

Os autores declaram não haver qualquer tipo de conflito de interesse neste trabalho.

\section{REFERÊNCIAS}

1. Heintze SD, Rousson V, Hickel R. Clinical effectiveness of direct anterior restorations: a meta-analysis. Dent Mater J. 2015;31(5):481-95. doi: 10.1016/j.dental.2015.01.015

2. Yap AU, Pandya M, Toh WS. Depth of cure of contemporary bulk-fill resin-based composites. Dent Mater J. 2016;35(3):503-10. doi: 10.4012/dmj.2015-402

3. Alkhudhairy FI. The effect of curing intensity on mechanical properties of different bulk-fill composite resins. Clin Cosmet Investig Dent. 2017;9:1-6. doi: 10.2147/CCIDE.S130085

4. Shimokawa CA, Turbino ML, Harlow JE, Price HL, Price RB. Light output from six battery operated dental curing lights. Mater Sci Eng C Mater Biol Appl. 2016;69:1036-42. doi: 10.1016/j.msec.2016.07.033

5. Nassar HM, Ajaj R, Hasanain F. Efficiency of light curing units in a government dental school. J Oral Sci. 2018;60(1):142-6. doi: 10.2334/josnusd.17-0071

6. Sakaguchi RL, Douglas WH, Peters MC. Curing light performance and polymerization of composite restorative materials. J Dent. 1992;20(3):183-8. doi: 10.1016/0300-5712(92)90136-z

7. Kovarik RE, Ergle JW. Fracture toughness of posterior composite resins fabricated by incremental layering. J Prosthet Dent. 1993;69(6):557-6o. doi: 10.1016/o022-3913(93)90280-2

8. Fronza BM, Rueggeberg FA, Braga RR, Mogilevych B, Soares LE, Martin AA, et al. Monomer conversion, microhardness, internal marginal adaptation, and shrinkage stress of bulk-fill resin composites. Dent Mater. 2015;31(12):1542-51. doi: 10.1016/j.dental.2015.10.001

9. Soygun K, Unal M, Ozer A, Gulnahar E, Bolayır G. Effects of different curing unites on bulk fill composites. Int J Oral Dent Health. 2015;1:13. doi: 10.23937/2469-5734/1510013

10. Alshali RZ, Salim NA, Satterthwaite JD, Silikas N. Post-irradiation hardness development, chemical softening, and thermal stability of bulk-fill and conventional resin-composites. J Dent. 2015;43(2):209-18. doi: 10.1016/j.jdent.2014.12.004

11. Marovic D, Tauböck TT, Attin T, Panduric V, Tarle Z. Monomer conversion and shrinkage force kinetics of low-viscosity bulkfill resin composites. Acta Odontol Scand. 2015;73(6):474-80. doi: 10.3109/00016357.2014.992810 
12. Abbas G, Fleming GJ, Harrington E, Shortall AC, Burke FJ. Cuspal movement and microleakage in premolar teeth restored with a packable composite cured in bulk or in increments. $J$ Dent. 2003;31(6):437-44. doi: 10.1016/so300-5712(02)00121-5

13. Alqudaihi FS, Cook NB, Diefenderfer KE, Bottino MC, Platt JA. Comparison of internal adaptation of bulk-fill and increment-fill resin composite materials. Oper Dent. 2019;44(1):E32-E44. doi: 10.2341/17-269-L

14. Do T, Church B, Veríssimo C, Hackmyer SP, Tantbirojn D, Simon JF, et al. Cuspal flexure, depth-of-cure, and bond integrity of bulk-fill composites. Pediatr Dent. 2014;36(7):468-73.

15. Hirata R, Kabbach W, Andrade OS, Bonfante EA, Giannini M, Coelho PG. Bulk fill composites: an anatomic sculpting technique. J Esthet Restor Dent. 2015;27(6):335-43. doi: 10.1111/jerd.12159

16. Al-Nabulsi M, Daud A, Yiu C, Omar H, Sauro S, Fawzy A, et al. Co-blend application mode of bulk fill composite resin. Materials (Basel). 2019;12(16):2504. doi: 10.3390/ma12162504

17. Kim EH, Jung KH, Son SA, Hur B, Kwon YH, Park JK. Effect of resin thickness on the microhardness and optical properties of bulk-fill resin composites. Restor Dent Endod. 2015;40(2):128-35. doi: 10.5395/rde.2015.40.2.128

18. Engelhardt F, Hahnel S, Preis V, Rosentritt M. Comparison of flowable bulk-fill and flowable resin-based composites: an in vitro analysis. Clin Oral Investig. 2016;20(8):2123-30. doi: 10.1007/s00784-015-1700-4

19. Papadogiannis D, Tolidis K, Gerasimou P, Lakes R, Papadogiannis Y. Viscoelastic properties, creep behavior and degree of conversion of bulk fill composite resins. Dent Mater. 2015;31(12):1533-41. doi: 10.1016/j.dental.2015.09.022

20. Ilie N, Bucuta S, Draenert M. Bulk-fill resin-based composites: an in vitro assessment of their mechanical performance. Oper Dent. 2013;38(6):618-25. doi: 10.2341/12-395-L

21. Fabianelli A, Sgarra A, Goracci C, Cantoro A, Pollington S, Ferrari M. Microleakage in class II restorations: open vs closed centripetal build-up technique. Oper Dent. 2010;35(3):308-13. doi: 10.2341/09-128-L

22. Roggendorf MJ, Krämer N, Appelt A, Naumann M, Frankenberger R. Marginal quality of flowable 4-mm base vs. conventionally layered resin composite. J Dent. 2011;39(10):643-7. doi: 10.1016/j.jdent.2011.07.004

23. Kelić K, Matić S, Marović D, Klarić E, Tarle Z. Microhardness of bulk-fill composite materials. Acta Clin Croat. 2016;55(4):607-14. doi: 10.20471/acc.2016.55.04.11
24. Rocha MG, Oliveira D, Correa IC, Correr-Sobrinho L, Sinhoreti M, Ferracane JL, et al. Light-emitting diode beam profile and spectral output influence on the degree of conversion of bulk fill composites. Oper Dent. 2017;42(4):418-27. doi: 10.2341/16-164-L

25. Shawkat ES, Shortall AC, Addison O, Palin WM. Oxygen inhibition and incremental layer bond strengths of resin composites. Dent Mater. 2009;25(11):1338-46. doi: 10.1016/j.dental.2009.06.003

26. Farahat F, Daneshkazemi AR, Hajiahmad Z. The effect of bulk depth and irradiation time on the surface hardness and degree of cure of bulk-fill composites. J Dent Biomater. 2016;3(3):284-91.

27. Moszner N, Fischer UK, Ganster B, Liska R, Rheinberger V. Benzoyl germanium derivatives as novel visible light photoinitiators for dental materials. Dent Mater. 2008;24(7):901-7. doi: 10.1016/j.dental.2007.11.004

28. Bucuta S, Ilie N. Light transmittance and micro-mechanical properties of bulk fill vs. conventional resin based composites. Clin Oral Investig. 2014;18(8):1991-2000. doi: 10.1007/ s00784-013-1177-y

29. Garoushi S, Vallittu P, Shinya A, Lassila L. Influence of increment thickness on light transmission, degree of conversion and micro hardness of bulk fill composites. Odontology. 2016;104(3):291-7. doi: 10.1007/s10266-015-0227-0

30. Chesterman J, Jowett A, Gallacher A, Nixon P. Bulk-fill resinbased composite restorative materials: a review. Br Dent J. 2017;222(5):337-44. doi: 10.1038/sj.bdj.2017.214

31. Bouschlicher MR, Rueggeberg FA, Wilson BM. Correlation of bottom-to-top surface microhardness and conversion ratios for a variety of resin composite compositions. Oper Dent. 2004;29(6):698-704.

32. Aranha AM, Giro EM, Hebling J, Lessa FC, Costa CA. Effects of light-curing time on the cytotoxicity of a restorative composite resin on odontoblast-like cells. J Appl Oral Sci. 2010;18(5):461-6. doi: 10.1590/s1678-77572010000500006

33. Salehi S, Gwinner F, Mitchell JC, Pfeifer C, Ferracane JL. Cytotoxicity of resin composites containing bioactive glass fillers. Dent Mater. 2015;31(2):195-203. doi: 10.1016/ j.dental.2014.12.004

34. Toh WS, Yap AU, Lim SY. In vitro biocompatibility of contemporary bulk-fill composites. Oper Dent. 2015;40(6):644-52. doi: 10.2341/15-059-L 
35. Son SA, Roh HM, Hur B, Kwon YH, Park JK. The effect of resin thickness on polymerization characteristics of siloranebased composite resin. Restor Dent Endod. 2014;39(4):310-8. doi: https://doi.org/10.5395/rde.2014.39.4.310

36. Hahnel S, Dowling AH, El-Safty S, Fleming GJ. The influence of monomeric resin and filler characteristics on the performance of experimental resin-based composites (RBCs) derived from a commercial formulation. Dent Mater. 2012;28(4):416-23. doi: https://doi.org/10.1016/j.dental.2011.11.016

37. Besegato JF, Jussiani EI, Andrello AC, Fernandes RV, Salomão FM, Vicentin BLS, et al. Effect of light-curing protocols on the mechanical behavior of bulk-fill resin composites. J Mech Behav Biomed Mater. 2019;90:381-7. doi: 10.1016/j.jmbbm.2018.10.026

38. AlShaafi MM, AlQussier A, AlQahtani MQ, Price RB. Effect of mold typeand diameteronthedepth of cure of threeresin-based composites. Oper Dent. 2018;43(5):520-9. doi: https://doi.org/10.2341/17-122-L
39. Price RB, Rueggeberg F, Harlow J, Sullivan B. Effect of mold type, diameter, and uncured composite removal method on depth of cure. Clin Oral Investig. 2016;20(7):1699-707. doi: https://doi.org/10.1007/s00784-015-1672-4

40. Al-Nahedh HN, AlawamiZ. Fracture resistanceand marginal adaptation of capped and uncapped bulk-fill resin-based materials. Oper Dent. 2020;45(2):E43-E56. doi: https://doi.org/10.2341/17-367-L

41. Oliveira D, Rocha MG, Correr AB, Ferracane JL, Sinhoreti M. Effect of beam profiles from different light emission tip types of multiwave light-emitting diodes on the curing profile of resin-based composites. Oper Dent. 2019;44(4):365-78. doi: https://doi.org/10.2341/16-242

42. Shimokawa CAK, Turbino ML, Giannini M, Braga RR, Price RB. Effect of light curing units on the polymerization of bulk fill resin-based composites. Dent Mater. 2018;34(8):1211-21. doi: https://doi.org/10.1016/j.dental.2018.05.002 